\title{
Modèles de raisonnement en conduite de cultures et conséquences pour les systèmes d'aide à la décision
}

\author{
JP Rellier 1, JC Marcaillou 2 \\ 1 INRA, Laboratoire d'intelligence artificielle, BP 27, 31326 Castanet-Tolosan Cedex; \\ ${ }^{2}$ École supérieure d'agriculture de Purpan, Voie du TOEC, 31076 Toulouse Cedex, France
}

(Reçu le 28 août 1989; accepté le 2 mai 1990)

\begin{abstract}
Résumé - L'aide à la décision technique ne consiste pas seulement à informer le décideur, à diagnostiquer l'état des cultures ou prévoir leur évolution, mais aussi à accompagner l'agriculteur dans sa propre démarche d'intégration de ces différents éléments. Un système informatique d'aide à la décision doit alors contenir un modèle des raisonnements mis en œuvre par l'agriculteur. À partir d'observations par entretiens de quelques processus de décision, on relève quelques caractéristiques structurelles communes à ces raisonnements. On en conclut qu'il est possible de modéliser les processus de décision. De tels modèles sont utilisables dans un but cognitif (pour représenter ce qu'on connaît d'un processus de décision), prévisionnel (pour étudier les propriétés de tel ou tel processus de décision), ou décisionnel (comme module d'un système informatique). L'apport potentiel de l'intelligence artificielle en la matière est souligné.
\end{abstract}

agriculture / aide à la décision / modélisation / raisonnement / système informatique

Summary - Models of agricultural reasoning and consequences on the design of decision support systems. Agricultural technical decision support should be used not only to inform the decision-maker, diagnose the state of the crops, or forecast their evolution, but also to accompany the farmer in his own process of integration of these elements. A computer-based decision support system should therefore contain a model of the reasoning carried out by the farmer. The present work attempts to describe and analyse this reasoning. It is based on a series of interviews with 18 farmers (the characteristics of the corresponding cropping systems are in table I, the interview framework in table II). The first section of the paper reports on the 2 types of behavior observed when the farmer is confronted with a technical problem (ie damaged soil structure): he either tries to find out and eliminate the cause of the emergence of the problem or only treats its observed consequences (fig 1). Following this, some common structural characteristics of the reasoning are outlined, leading to the feasibility of modelling decision-making processes. Examples of such models are analysed in 2 particular areas: weeds infestation (fig 2) and the annual crop-plot assignment (fig 3). Some important consequences of the above-mentioned analysis on the designing of decision support systems (DSS) are the necessity for a DSS: to help the farmer in jointly using information processing, diagnosis and solution design facilities; to take into account the temporal and spatial dimensions of the considered decision-making process. In addition, we stress the need for specific research on decision-making processes modeling: 3 classes of models are now considered (fig 4). Decision-making process models may serve 3 purposes: cognitive (to represent what we know about a given decision making process), predictive (to investigate the properties of such and such decision process) and decisive (as part of a DSS). The potential contribution of artificial intelligence in this area is outlined.

agriculture / decision support / modeling / reasoning / computer system

\section{INTRODUCTION}

La production agricole dépend à la fois de l'effet des différentes composantes du milieu sur les peuplements végétaux ou les animaux, et de la façon dont l'agriculteur, par ses pratiques techniques, utilise, provoque ou inhibe cet effet.
Une pratique technique nécessite toujours, plus ou moins directement, une phase préalable de décision, c'est-à-dire un choix entre plusieurs alternatives, de façon à satisfaire un ou plusieurs objectifs. En agriculture comme dans d'autres domaines, la difficulté de ce choix peut venir du grand nombre d'alternatives, d'objectifs ou de paramètres à prendre en compte, ou de contraintes 
de temps imposées à la résolution du problème. De plus, les alternatives sont souvent connues de manière incomplète, et les connaissances sur la façon d'atteindre un objectif sont incertaines, conflictuelles ou incomplètes. Ainsi, une décision nécessite-t-elle fréquemment une phase d'élaboration des différentes alternatives possibles, et pas seulement une sélection parmi un ensemble de solutions pré-énumérées. L'aide à la décision vise à réduire l'ensemble de ces difficultés.

La conception des systèmes automatiques d'aide à la décision a jusqu'à présent été guidée par plusieurs courants. De la recherche opérationnelle et des travaux sur l'optimisation mathématique sont issus, entre autres, les algorithmes généraux de programmation dynamique et de programmation linéaire (France et Thornley, 1984). La première technique est utilisée pour trouver une solution optimale à des problèmes impliquant une séquence d'actions (par exemple, utilisation ou non d'un insecticide à une série de dates). La seconde recherche une combinaison d'activités qui maximise une fonction objectif en respectant des contraintes sur les ressources partagées par les activités. Dans le cadre de l'analyse de décision (decision analysis, en anglais) (Howard, 1968), la démarche consiste à décrire les connaissances générales relatives au domaine de la décision (par exemple, le choix d'une date de semis), mais aussi les connaissances relatives à la situation particulière du décideur, en termes de circonstances (facteurs environnementaux) et de préférences (facteurs personnels). La décision est ensuite prise sur la base normative de la théorie statistique de la décision (maximum de la fonction d'utilité, par exemple).

Remarquons que ces outils contiennent une méthode de résolution (l'optimisation d'une fonction) sur laquelle le décideur n'a d'autre liberté que de choisir de la mettre en œuvre ou non. Le concept de système interactif d'aide à la décision (Heurgon, 1985), ou de decision support system (Coulson et Saunders, 1988) étend l'intervention du décideur à l'identification du problème et à la recherche interactive de la voie de résolution. Cela suppose que le système puisse répondre, c'est-à-dire qu'il connaisse une gamme de chaînes de traitement des données, dans laquelle le décideur navigue en fonction de sa propre perception du problème à résoudre et de la situation qui l'a engendré. Les progrès réalisés sur l'interactivité des ordinateurs, sur la mise en cuvre des systèmes d'informations (bases de données, etc), ont favorisé l'intérêt pratique du concept.

Notre travail se situe dans un $3^{e}$ courant, celui de l'intelligence artificielle (IA). Si les premières tentatives en résolution de problème ont visé des procédures indépendantes du domaine de décision [par exemple le general problem solver ( $\mathrm{Ne}$ well et Simon, 1963), le développement de l'ingénierie de la connaissance a permis de coder des procédures de raisonnement dépendantes du domaine. En particulier, les systèmes experts contiennent des connaissances empiriques difficilement représentables dans d'autres cadres et permettent ainsi de résoudre des problèmes pour lesquels on ne connaît pas d'algorithme efficace. Une voie de recherche s'ouvre actuellement, visant une fertilisation mutuelle entre les concepts et les outils élaborés dans les 3 courants (Richter, 1988).

Dans ce contexte, le point de départ de notre étude est le suivant. Pour qu'un agriculteur, face à un problème particulier de décision, accepte l'aide d'un système informatique, il faut que ce dernier contienne le modèle d'un raisonnement acceptable. Ce raisonnement peut être conçu par un ou plusieurs spécialistes du domaine; on construit alors un système expert dont il convient de tester l'acceptabilité. C'est la voie empruntée, par exemple, par Le Corfec (Le Corfec, 1988). La voie que nous explorons ici consiste à modéliser le raisonnement de l'agriculteur lui-même. Nous cherchons à décrire la façon dont l'agriculteur intervient dans la formulation et le processus de résolution de son problème de décision, et à formaliser cette description par des modèles. Ces modèles sont ensuite utilisés pour prévoir ce que devrait être le comportement et les fonctionnalités correspondantes d'un système informatique d'aide à la décision.

\section{INDICATIONS MÉTHODOLOGIQUES}

Notre étude* (Marcaillou, 1988) porte sur un échantillon de 18 agriculteurs, appartenant à 9 groupes de développement agricole. Leurs systèmes de production sont fondés sur les grandes cultures, installées sur une large gamme de surfaces et de terrains. Quelques descripteurs de l'échantillon sont reportés dans le tableau I.

\footnotetext{
* Réalisée avec le concours de la Fédération nationale des groupes d'études et de développement agricoles (FNGEDA) et de la Station d'agronomie de l'INRA à Toulouse.
} 
La première exigence étant de recueillir, non pas des données objectives sur les actions techniques de l'agriculteur, mais plutôt sa démarche intellectuelle vers l'action, nous avons exclusivement analysé le contenu d'entretiens. Nous avons choisi la technique de l'entretien non directif de recherche (Rogers, 1945; Blanchet, 1985), qui favorise l'expression de l'interlocuteur (quantitativement et sur les types de réponses) et qui limite les risques d'influence et d'orientation de la part des interviewers.

Nous avons considéré que l'étude du processus de décision d'un agriculteur serait plus fructueuse si elle portait sur une préoccupation actuelle de l'agriculteur. Comme nous ne pouvions prévoir ces préoccupations, nous leur avons consacré une première phase d'entretiens (voir en tableau II un extrait du guide d'entretien). Pour l'étude plus fine des processus de décision, une deuxième série d'entretiens a ensuite été menée sur 2 thèmes souvent évoqués dans la $1^{\text {re }}$ phase. Pour le premier de ces thèmes (la lutte contre les adventices), nous avons demandé à l'agriculteur de se remémorer tous les aspects du problème, de la première alarme jusqu'au jugement de la situation actuelle, et d'établir les liens (chronologiques, causaux, etc) entre ces éléments. Pour le $2^{e}$ thème (mise en place de l'assolement), nous avons demandé à l'agriculteur de dessiner schématiquement mais complètement le parcellaire avec l'assolement en cours (1986-1987), puis de raisonner devant nous, sur son dessin, l'assolement de la prochaine campagne.

Préalablement aux entretiens, mais aussi au cours de l'étude, nous avons recherché et affiné des outils d'analyse méthodique et rigoureuse de leur contenu : retranscription quasi-intégrale des enregistrements, inventaire des unités de sens (mot, phrase ou partie du discours présentant une unité thématique), analyse de la cohérence du discours, recherche du référentiel de l'agriculteur, reconnaissance des types de raisonnements selon une typologie pré-établie, normalisation de la représentation des dynamiques de raisonnement (Marcaillou, 1988).

\section{QUELQUES OBSERVATIONS SUR LE DÉCIDEUR}

\section{Une typologie des problèmes de conduite technique des cultures}

Nous avons repéré, dans le discours de l'agriculteur, que celui-ci attribuait dans certains cas la responsabilité d'un problème technique à une cause : par exemple, si telle infestation d'adventices est due, selon lui, à la répétition des mêmes cultures, il parle de problème d'assolement. Ces imputations de responsabilité permet-

Tableau I. Quelques descripteurs de l'échantillon d'agriculteurs. LA : limono-argileux; L : limoneux; B : boulbènes; $A C$ : argilo-calcaire; $T$ : terrefort; all : alluvions. UTH : $n+1$ indique $n$ personnes permanentes plus une occasionnelle.

\section{Agric Type de sol SAU (ha) UTH Cult été (\%) Cult principales Autres activités Jeune agric}

\begin{tabular}{|c|c|c|c|c|c|c|c|}
\hline$A_{1}$ & LA & 30 & 1 & 100 & maiis, tournesol & 1 & / \\
\hline $\mathrm{A}_{2}$ & alluvions & 40 & $t+$ & 100 & soja & arboriculture & 1 \\
\hline$A_{3}^{2}$ & $\mathrm{~B}$ & 56 & $1+$ & 100 & maïs, soja & volaille & I \\
\hline$A_{4}^{3}$ & $\mathrm{~B}$ & 73 & 2 & 90 & maïs, soja & maraîchage & I \\
\hline$A_{5}^{4}$ & $L$ & 40 & 1 & 70 & maïs, colza & tabac & oui \\
\hline$A_{6}$ & alluvions & 140 & $2+$ & 70 & maïs, céréales & viande, bovins & 1 \\
\hline$A_{7}^{0}$ & LA & 74 & $1+$ & 75 & maïs, blé & 1 & / \\
\hline$A_{8}^{\prime}$ & B & 85 & 3 & 70 & maïs & bovins-viande & I \\
\hline$A_{9}^{\circ}$ & $\mathrm{B}$ (coteaux) & 78 & $1+$ & 65 & maïs, blé & 1 & 1 \\
\hline$A_{10}$ & all, $A C, B$ & 98 & $2+$ & 60 & maïs, céréales & viande-bov, ovin & oui \\
\hline$A_{11}$ & $A C$ & 142 & $2+$ & 42 & blé, maïs & 1 & 1 \\
\hline$A_{12}$ & $\mathrm{~T}$, all & 45 & 1 & 35 & blé, tournesol & I & oui \\
\hline$A_{13}$ & $A C, B$ & 85 & $2+$ & 55 & céréales, tournesol & porc & oui \\
\hline$A_{14}^{10}$ & $A C, B$ & 52 & $1+$ & 46 & céréales, soja & I & 1 \\
\hline$A_{15}^{74}$ & $A C$ & 62 & 1 & 25 & blé, colza & oie & I \\
\hline$A_{16}^{10}$ & $A C$ & 120 & $1+$ & 20 & blé, tournesol & I & I \\
\hline$A_{17}^{16}$ & $B, A C$ & 190 & 3 & 33 & blé, colza & volaille & I \\
\hline$A_{18}$ & $A C$ & 64 & $1+$ & 10 & blé, colza & I & 1 \\
\hline
\end{tabular}


Tableau II. Extrait du guide d'entretien utilisé lors de la première phase de l'étude.

Guide d'entretien

1) Présentation

2) Analyse de l'existant

3) Évolution de l'exploitation

4) Quels sont les problèmes rencontrés en matière de conduite technique (culture et sole)

4-1) Énoncé des problèmes

But :

Connaître les problèmes dans les termes utilisés par l'agriculteur; reconnaître la hiérarchie établie par l'agriculteur Pourquoi?

- Pour pouvoir, à partir du discours de l'agriculteur, déceler le niveau d'imputation du problème :

1) la sole, 2) la parcelle, 3) la culture, 4) la plante, 5) une opérations culturale, 6) un stade, 7) une méconnaissance

- trouver d'éventuels niveaux d'imputation communs à plusieurs exploitations

\section{4-2) Pourquoi est-ce un problème ?}

But 1 :

Situer la cause du problème : 1) contrainte de structure (drainage parcelle), 2) contrainte de moyens, 3) manque de données, d'informations, 4) crainte due à un risque important

But 2:

Trouver la raison de l'émergence en bonne place : 1) difficulté de résolution du problème, 2) conséquences importantes du problème au niveau de l'exploitation (le problème n'est pas forcément difficile, mais on n'a pas droit à l'erreur)

But :

4-3) Pour chaque problème, comment l'agriculteur s'en est-il rendu compte ?

L'alarme est-elle d'origine 1) interne (essais, résultats d'analyses, 2) externe (information, rencontre),

si l'alarme est d'origine externe, provient-elle 1) de techniciens, 2) de revues spécialisées, 3) d'autres agriculteurs ?

Voir ici s'il y a un problème important qui «ressort" souvent. Si ce problème est imputé souvent à un même niveau.

tent de distinguer dans notre échantillon 2 types d'expression d'un problème (fig 1).

Le $1^{\text {er }}$ correspond au niveau central de la figure. Dans ce cas, l'agriculteur accepte l'existence de l'anomalie et ne considère que les effets, qu'il combat dans leurs manifestations et non dans leurs causes : une infestation d'adventices entraîne un désherbage chimique, une structure de sol dégradée entraîne un décompactage, etc. Le second type correspond aux niveaux externes (haut et bas) de la figure. L'agriculteur ne considère pas seulement les effets. La recherche des causes fait remonter le problème vers une classe externe, et l'agriculteur envisage d'autres solutions que celle de la classe centrale originelle. Les solutions sont alors plutôt préventives : par exemple, une prolifération d'adventices en monoculture entraîne l'introduction d'une autre culture permettant une rotation.

Un niveau d'expression directe dans une classe centrale implique un raisonnement ciblé, sur les désherbants par exemple, avec application de recettes et association d'un remède à un mal. Un niveau d'expression dans une classe externe implique un raisonnement analytique pour

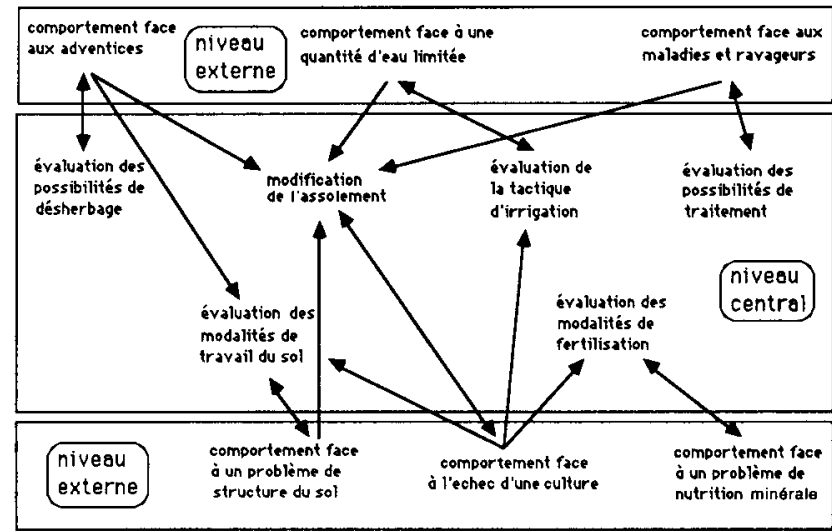

Fig 1. Typologie des problèmes de conduite technique des cultures. Une flèche simple indique une voie possible pour résoudre un problème posé au niveau externe; une flèche double illustre, dans un sens, une voie possible de résolution, dans l'autre sens, la remontée du problème vers le niveau externe. 
lequel les classes centrales constituent autant de voies permettant de réagir.

Cette partie préalable de l'étude a souligné la spécificité des questions soulevées par les agriculteurs : c'est-à-dire qu'elles qui portent, non pas sur la con-ception d'ensemble de la conduite des cultures (par exemple en termes d'itinéraire technique), mais sur des épisodes de la conduite. En fait, les agriculteurs ont associé des problèmes à des anomalies face auxquelles ils ont (ou ont eu) à réagir. Mais surtout, elle a montré qu'on ne pouvait pas associer à la nature objective d'un problème (qui se manifeste par des symptômes mesurables) un seul type de raisonnement : c'est l'individu qui oriente pleinement la résolution, en particulier par son diagnostic. $\mathrm{Ce}$ point sera illustré par la suite.

\section{Description des processus de prise de décision}

Nous avons choisi de développer 2 problèmes souvent évoqués par les agriculteurs de notre échantillon. Le $1^{\text {er }}$ est celui de la lutte contre les adventices, quand il est posé à un niveau externe (fig 1); le second est celui de la mise en place de l'assolement, qui est posé au niveau central.

\section{Processus de décision face aux adventices}

De façon globale, ce processus se caractérise par un cycle se répétant chaque année de la façon suivante :

- constitution d'un diagnostic induit par les éléments ayant révélé le problème : l'agriculteur identifie plus ou moins complètement le problème, à partir d'observations et de connaissances;

- recherche de solutions : c'est la phase active du raisonnement, elle débouche sur une action;

- contrôle des résultats (il s'agit d'évaluer le résultat de l'action entreprise); pour cela, les éléments de diagnostic (observations) font l'objet d'un nouvel examen; en cas de constat d'échec, il y a recherche de nouvelles solutions.

On remarque qu'aucune de ces phases n'est répétée sans qu'il y ait eu entre temps passage à l'action. Nous parlerons de raisonnement par étapes pour caractériser cette démarche. La figure 2 en donne un exemple (agriculteur $A_{10}$ dans le tableau I).
Les éléments de diagnostic font apparaître une alarme et, par là-même, induisent la perception d'un événement sous forme d'un problème. De ces éléments est inféré un diagnostic dont la formulation sous-tend l'orientation future du raisonnement. L'examen des éléments de diagnostic amène les remarques suivantes. L'aspect visuel prédomine : l'infestation des adventices est évaluée globalement à partir de taches, de ronds; à aucun moment il n'y a de comptage réalisé, ni de définition d'un taux d'infestation. II n'y a pas d'expression de notions relatives au rendement et peu d'idées sur les pertes occasionnées par l'accident. L'alarme est déclenchée par la vitesse de propagation des adventices plutôt que par le niveau actuel de l'infestation. L'agriculteur imagine, de façon qualitative, les conséquences à terme de cette évolution : nous qualifions ce raisonnement de simulatif. En fonction du diagnostic s'élabore un raisonnement que nous qualifions d'analytique, au sens où il y a évaluation des facteurs de causalité, et où, de plus, le raisonnement s'appuie sur de nombreuses notions propres à l'individu (sur l'application des désherbants, sur telle ou telle culture, etc).

Ce qui caractérise la résolution de ces problèmes, posés à un niveau externe, c'est la mise en œuvre de solutions ne se situant pas unique-

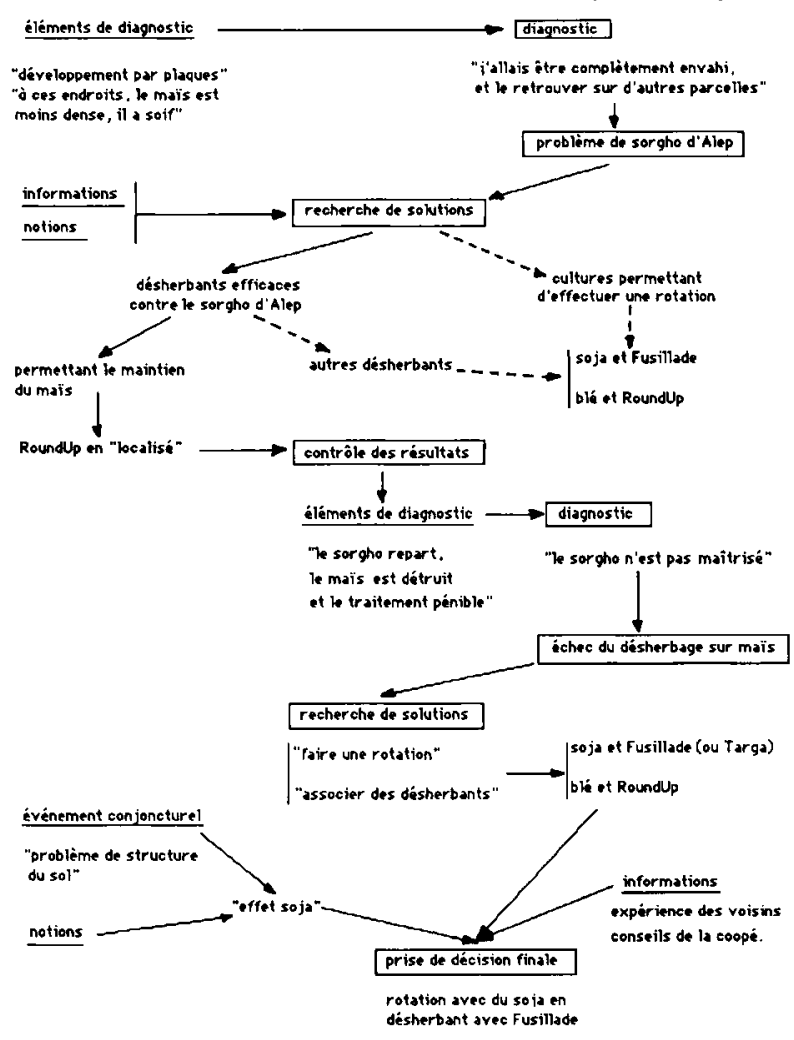

Fig 2. Exemple de raisonnement de lutte contre une infestation d'adventices. Une flèche discontinue signale une proposition évaluée mais ne correspondant pas à une action de l'agriculteur. 
ment dans la classe centrale d'imputation naturelle. Ce qui est encore plus remarquable, c'est l'association de classes centrales dans la résolution du problème. Ainsi, dans l'exemple de la figure 2, l'agriculteur associe une rotation et un désherbage sur la culture introduite.

Enfin, soulignons l'importance d'événements conjoncturels lors de la prise de décision. Dans l'exemple, nous voyons qu'un choix s'est présenté entre les associations blé-RoundUp et sojaFusillade. La décision a été largement conditionnée par le problème de structure du sol rencontré antérieurement et qui a entraîné le choix du soja.

Le contrôle des résultats s'effectue par un raisonnement comparatif. Les éléments de diagnostic sont reconsidérés pour cette étape et comparés à la situation initiale; pour ce faire, l'agriculteur attend une situation identique : dans notre exemple, il attend le retour du maïs pour se prononcer définitivement sur l'efficacité de sa solution.

\section{Processus de décision pour la mise en place de l'assolement}

La modification de l'assolement peut être une solution à des problèmes d'abord exprimés à un autre niveau par l'agriculteur (problème de lutte contre les adventices, par exemple). Cependant, même quand elle n'est pas motivée par la résolution d'un problème particulier, la mise en place de l'assolement est un acte nécessairement réalisé chaque année. Nous avons adopté ce second point de vue dans la conduite de l'entretien.

Mettre en place un assolement consiste à affecter une culture à chacune des parcelles cultivables de l'exploitation. Nous ne reprendrons pas ici l'analyse des éléments pris en compte par l'agriculteur dans cette opération [évaluation des cultures, des parcelles, considérations sur les travaux, etc (Marcaillou, 1988)]. Nous examinerons les raisonnements lors de l'affectation proprement dite, à l'aide d'un exemple. On désignera ici par le mot sole un ensemble de parcelles présentant une caractéristique commune (irrigation, éloignement, secteur en coteaux, etc) qui justifie de les considérer conjointement dans le même processus d'affectation *.

Nous avons identifié, dans notre échantillon, deux types d'affectation:
- les affectations résultant d'une prise en considération de divers facteurs (en particulier agronomiques) au niveau de la parcelle, des cultures, etc : nous l'appelons affectation considérative (les aspects économiques n'en sont pas le déterminant direct ni principal);

- les affectations résultant avant tout, d'une volonté de l'agriculteur, d'un objectif déterminé pour sa sole; peu de préoccupations agronomiques interviennent alors; nous qualifions cette affectation d'intentionnelle. Les cas de figure principaux sont les cultures à intérêt économique important et les cultures pour lesquelles l'agriculteur mène des essais personnels.

La figure 3 représente un des processus d'affectation observés. La démarche de cet agriculteur $\left(A_{15}\right.$ dans le tableau $\left.I\right)$ dépend largement de la notion de sole irrigable. Sa surface est de 45 ha divisés en 3 blocs de 15 ha chacun. Le potentiel d'irrigation est de 15 ha (lac collinaire). La culture irriguée (maiis) est généralement répartie alternativement sur 2 blocs (1 et 2). Le bloc 3 est ordinairement délaissé pour le maïs, car la pente y est jugée excessive. Les 15 ha de maïs sont jugés indispensables par l'agriculteur pour des raisons économiques. Cela entraîne une affectation intentionnelle du maïs sur les blocs 1 et 2 . Le seul facteur agronomique considéré est la notion de précédent cultural, justifiant pour cette sole une rotation maïs-blé. On remarque cependant un contrôle relatif à la structure du sol, qui peut être dégradée par une récolte du maïs en condition humide. Dans ce cas, l'agriculteur refuse de revenir au bout d'un an à une culture irriguée. La succession maïs-blé devient maïs-blé-

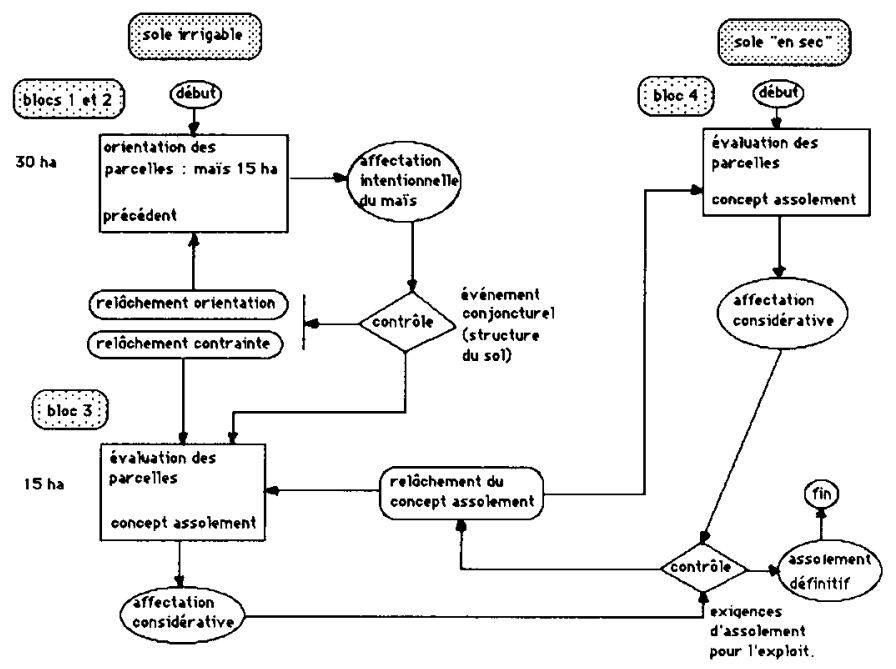

Fig 3. Exemple de modèle de raisonnement lors de la mise en place de l'assolement.

\footnotetext{
" Dans une autre acception, non retenue ici, la sole désigne l'ensemble des parcelles consacrées une année donnée à la même culture (la sole de blé).
} 
colza-maïs, avec un relâchement de l'orientation maïs de ces 2 blocs. Dans le même temps l'agriculteur reconsidère son évaluation des parcelles du bloc 3 (succession blé-colza, en général), relâche la contrainte pente, et y affecte sa culture de maiis irrigué, en totalité ou en partie. Ainsi, l'affectation des parcelles du bloc 3 dépend de l'affectation dans les blocs 1 et 2 .

L'affectation dans la sole "en sec" (bloc 4, 15 ha) est indépendante des 2 autres. C'est une affectation considérative, pas très développée, l'agriculteur considérant l'éventail des cultures possibles comme très limité (blé, colza et accessoirement tournesol).

On remarque un contrôle final sur les exigences d'assolement pour l'exploitation. En effet, l'agriculteur ne tient pas à attribuer une trop grande surface au colza. Or, en cas de problème de structure du sol, la surface en colza est susceptible de doubler. C'est un facteur pouvant favoriser l'implantation du tournesol.

Cet exemple montre qu'il peut exister, sur une même exploitation, plusieurs processus d'affectation. Nous avons distingué :

- les processus dépendants, où l'agriculteur ne s'intéresse à l'affectation sur une sole que lorsque l'affectation sur telle autre sole a déjà été réalisée; généralement le même type d'affectation (considérative) est utilisé sur les processus dépendants, les facteurs considérés sont simplement différents;

- les processus indépendants, où les affectations respectives se réalisent sans qu'il y ait prise en compte de l'affectation réalisée par ailleurs. On observe seulement quelquefois un contrôle final pour ces différentes entrées.

$\mathrm{Ce}$ qui distingue le raisonnement de l'assolement de celui associé à la lutte contre les adventices, c'est qu'on observe dans le $1^{\mathrm{er}}$, préalablement à toute action, une étape de contrôle au terme de laquelle l'agriculteur peut revenir sur les affectations déjà envisagées, pour affiner sa décision. Nous parlons ici de raisonnement itératif. Cette étape de contrôle semble obligatoire pour les affectations considératives. En effet, l'agriculteur se place alors principalement au niveau de la parcelle. De ce fait, il peut arriver que l'intérêt de couples parcelle-culture fasse envisager un ensemble d'affectations en désaccord avec les exigences d'assolement pour l'exploitation. $\mathrm{Ce}$ contrôle a des conséquences directes de 2 types :

- un relâchement des contraintes parcellaires : l'agriculteur abandonne les considérations agronomiques, de telle sorte que les plus fortes contraintes au niveau de l'exploitation deviennent satisfaites (cas de l'affectation considérative); par exemple, à cause d'une surface en blé à ne pas dépasser (contrainte de stockage), l'agriculteur affecte un pois à une parcelle qu'il juge pourtant trop pentue;

- plus rarement, un relâchement de l'objectif assigné par l'agriculteur à une sole (cas de l'affectation intentionnelle).

Certaines affectations sont temporairement conditionnelles. II s'agit de parcelles pour lesquelles l'agriculteur n'est pas clairement décidé; il émet alors une opinion sous condition, ou affecte 2 cultures à la même parcelle, en reportant sa décision à plus tard. L'affectation définitive se réalise, soit lors de l'arrivée d'informations nouvelles, soit au cours de la phase itérative du raisonnement, lorsque l'agriculteur évalue de nouveau ses considérations en vue d'affiner son raisonnement. Ces parcelles sont utilisées pour harmoniser l'assolement global et jouent un rôle tampon relativement au contrôle des exigences d'assolement au niveau de l'exploitation.

\section{CONSÉQUENCES POUR LA CONCEPTION DE SYSTĖMES D'AIDE À LA DÉCISION}

Les observations précédentes n'ont pas de portée générale, au sens où les problèmes évoqués par les agriculteurs que nous avons rencontrés et les solutions apportées auraient pu être différents sur un autre échantillon. Cependant, audelà des particularités de chaque cas (par exemple, tel ou tel élément de diagnostic pour un problème donné), nous leur avons trouvé des points communs qui permettent de développer, de façon non exhaustive, 4 idées sur la conception des systèmes informatique d'aide à la décision.

\section{La nécessaire intégration de différents niveaux d'aide}

Le premier niveau d'aide consiste à fournir au décideur l'information qui lui semble utile. Deux classes d'informations sont concernées :

- les informations sur l'environnement de l'exploitation (données économiques, produits commercialisés, prévisions météo, etc), et

- les données relatives à l'exploitation ellemême (passé cultural des parcelles, résultats technico-économiques). Cela relève d'outils très 
classiques de traitement de l'information (accès, tris, mises à jour) et de traitement de données numériques (calculs). Leur mise en œuvre est cependant problématique : qui sera maître d'œuvre, au niveau de chaque région ou petite région, des banques de données? Comment choisir et valoriser les données (potentiellement très nombreuses) recueillies par l'agriculteur, pour ne pas le décourager de le faire? Enfin, nous avons constaté que le référentiel de l'agriculteur englobe souvent d'autres confrères situés dans des conditions identiques, ou réputés compétents. Ces informations ne relèvent d'aucune des 2 classes distinguées plus haut. Et de façon générale, il paraît difficile de délimiter l'ensemble des éléments sur lesquels l'agriculteur fonde ses décisions. En conséquence, certaines décisions ne pourront pas être inférées par le système, mais devront être proposées par l'agriculteur au système, pour qu'il les évalue par exemple.

Le $2^{2}$ niveau d'aide en conduite des cultures, porte sur la compréhension et la prévision du comportement des cultures. La compréhension passe par un diagnostic, c'est-à-dire une caractérisation d'une situation observée plus une identification des causes d'écart à une situation prévue. La prévision relève de simulations, c'est-àdire d'hypothèses faites sur différents facteurs du comportement ultérieur des cultures. Nous avons constaté que les diagnostics des agriculteurs sont souvent comparatifs, par rapport à des situations voisines ou d'un proche passé ("sur les plaques de sorgho d'Alep, le maïs est moins dense...»), et les prévisions intuitives («... j'allais être envahi, et le retrouver sur d'autres parcelles»). On pourra améliorer ces inférences en utilisant, lorsqu'ils existeront, des modèles des phénomènes concernés. On retrouve ici les connaissances superficielle et profonde, distinguées, dans le domaine des systèmes experts, par leur fondement ou non sur une représentation explicite des relations cause-conséquence (Pierret-Golbreich, 1989). Les modèles les plus rapidement disponibles porteront sur les processus du système plante-sol-climat (croissance, développement, alimentation, modification des états minéraux et hydrique du sol). Des modèles de systèmes plus complexes, faisant intervenir d'autres agents (adventices, maladies, prédateurs) sont aussi ébauchés. L'apport de la discipline agronomique sera déterminant à ce niveau d'aide : mise au point d'indicateurs pertinents pour le diagnostic, élaboration de modèles opérationnels pour la prévision.
Les systèmes d'aide à la décision doivent accéder à un $3^{\theta}$ niveau : celui qui vise à assister le décideur dans l'utilisation conjointe des informations, des diagnostics et des prévisions, en vue de la réalisation d'un objectif, c'est-à-dire à l'accompagner dans son processus décisionnel. Nos observations de l'agriculteur nous aident à mieux concevoir cette fonction, sur 2 points.

Le $1 \mathrm{er}$ point concerne l'identification du problème et le choix du raisonnement. La typologie des préoccupations nous a montré qu'on ne pouvait associer un seul type de raisonnement à la nature d'une question soulevée par un agriculteur. De ce fait, un système d'aide à la décision sera confronté aux 2 niveaux de positionnement distingués (fig 1 ) et il devra être capable, le cas échéant, de sortir de la classe centrale naturelle pour orienter l'agriculteur, s'il le souhaite, vers d'autres solutions. Par exemple, face à un problème d'adventices, il devra être en mesure d'évaluer concrètement un changement d'assolement. Le système devra alors évaluer comparativement plusieurs voies de résolution :

- évaluation d'un changement de rotation : différence de marges, évaluation de l'effet sur les adventices, calculs pour les divers désherbants associables, etc;

- évaluation du maintien de la culture : produits à associer, efficacités, surcoûts, etc;

- évaluation de la non-action : évolution de l'infestation, conséquences sur la culture.

Un système d'aide à la décision pour l'éradication des adventices qui ne communiquerait qu'un diagnostic et une liste de produits serait inadapté à la demande d'une partie de ses interlocuteurs. Pour capter l'attention des utilisateurs, un tel système doit présenter une certaine amplitude de résolution parmi les voies figurées par les différentes classes centrales (fig 1 ).

Le second point, développé dans les 2 paragraphes suivants, concerne la structure des raisonnements de l'agriculteur.

\section{LA DIMENSION TEMPORELLE DU RAISONNEMENT}

Nous avons relevé, sans prétendre là encore être exhaustifs, 2 types d'intervention du temps, correspondant aux raisonnements par étapes et itératif.

Le raisonnement par étapes est rythmé par les réactions du système biologique aux actions techniques réalisées et à l'environnement. Dans 
notre cas de lutte contre les adventices, on observe les résultats sur une campagne pour agir la campagne suivante. Le raisonnement par étapes accompagne l'action, il la prépare puis contrôle ses effets observés.

Le raisonnement itératif est rythmé par l'appréciation du décideur sur les qualités d'une action envisagée, relativement à un objectif. On l'a rencontré lors de la mise en place de l'assolement. II précède complètement l'action. Ce type de raisonnement se justifie doublement. D'une part, l'agriculteur a naturellement tendance à préparer ses décisions, même sans en avoir encore tous les éléments, et à les affiner après avoir pris connaissance d'éléments complémentaires, et d'autre part, cas typique de l'affectation des cultures, l'agriculteur raisonne d'abord sur une partie de son exploitation, en se réservant un contrôle ultérieur au niveau global.

Si on admet la nécessité, pour un système informatique, de reproduire des dynamiques de raisonnement pour être, non seulement un système fournissant des informations ponctuelles, mais un système accompagnant la décision, il faut alors trouver des solutions à certains problèmes de traitement et de gestion de connaissances (et plus seulement de données) : comment garder la mémoire d'étapes antérieures du raisonnement, entre consultations du système, pour comparer les diagnostics, acquérir de nouvelles connaissances par l'analyse des faits (apprentissage); comment garder la trace d'itérations antérieures pour reprendre partiellement un raisonnement, essayer une hypothèse alternative, introduire des données devenues plus précises, certaines ou complètes ?

\section{La concurrence des raisonnements}

Là encore, nos observations permettent de distinguer 2 cas. Des raisonnements parallèles sont conduits par l'agriculteur sur des parcelles différentes, mais en même temps, pour mettre en œuvre des solutions différentes au même problème. Dans un cas de lutte contre les adventices, non rapporté ici, l'agriculteur a testé, d'une part un produit curatif et, sur une autre parcelle, l'effet préventif d'une modification de la succession des cultures. Un système d'aide à la décision devrait alors aider l'agriculteur à comparer les 2 voies de résolution (comparaison des résultats, simulation de l'extension d'un choix à toutes les parcelles infestées). Ce point ne semble pas soulever de problèmes informatiques nouveaux, mais l'effort doit porter sur l'ergonomie et l'efficacité du système d'information (accès aux données, calculs, synthèses graphiques).

Un autre cas est celui du raisonnement de l'utilisation de ressources limitées sur l'exploitation. II faut alors raisonner des priorités d'intervention entre différentes parcelles (limite de ressource en eau, ou de main d'œuvre, par exemple). Dans le cas du raisonnement itératif d'affectation des cultures aux parcelles, l'agriculteur effectue un contrôle sur l'ensemble des parcelles, pour vérifier qu'une contrainte n'est pas dépassée.

On soulève ici le problème de la représentation des relations informationnelles entre les différentes entités matérielles de l'exploitation (parcelles, lieux de stockage, etc). L'agriculteur doit pouvoir raisonner séparément (concurremment) sur chaque entité (disons, chaque parcelle) comme il est naturel de le faire. Mais une transmission d'information entre entités (par exemple, entre parcelles et lieux de stockage circule un stock récolté) doit informer l'agriculteur de la nécessaire révision de certains raisonnements (par exemple, si la capacité de stockage d'un lieu est dépassée). Une représentation de type objets, associée à une programmation des relations sous forme de messages (Bailly et al, 1987), peut être envisagée pour résoudre ce problème. Elle permet de décrire une parcelle, par exemple, par une série de caractéristiques statiques (surface) et dynamiques (évolution du couvert végétal), mais aussi par son comportement vis-à-vis des autres entités : envoi d'un message lorsqu'une caractéristique est modifiée, traitement d'un message en provenance d'une autre entité. Cette programmation à la fois répartie et interdépendante nous semble un bon modèle de la façon dont l'agriculteur perçoit son exploitation.

D'autre part, comme dans la prise en compte de la dimension temporelle du raisonnement, nous retrouvons un cas de révision partielle d'un ensemble de conclusions, due à l'arrivée de nouvelles données. Ainsi, un choix (l'affectation d'une culture à une parcelle, par exemple) peut être considéré comme une hypothèse. Si cette hypothèse conduit à une incohérence avec une connaissance déjà établie (par exemple, dépassement d'une quantité maximale de stock), il faut essayer une hypothèse alternative, en prenant soin de conserver toutes les conclusions sauf celles relatives à l'hypothèse abandonnée. Les travaux de de Kleer (1986), qui fondent la technique des ATMS (assumption-based truth 
maintenance system), peuvent aider à formuler et à résoudre ce problème.

\section{L'extension du champ d'application de la modélisation}

Jusqu'à présent, dans le domaine qui nous concerne, on a essentiellement tenté de modéliser les systèmes biotechniques, qu'ils soient naturels (le peuplement végétal dans son environnement pédoclimatique et technique; Charpenteau et Rellier, 1980) ou organisationnels (un ensemble de contraintes liant des activités à mettre en œuvre à des ressources limitées; Marty et al, 1984). Une extension du champ d'application de la modélisation, déjà évoquée par Attonaty et Soler (1988), vise les systèmes décisionnels. Notons bien ici que les modèles que nous avons présentés (figs 1 et 2) sont une vision, non pas du système décisionnel, mais du processus de décision. Autrement dit, nous avons figuré, non pas les éléments du système décisionnel et leurs relations, mais le résultat du fonctionnement de ce système, c'est-à-dire l'enchaînement observé des raisonnements et des actions. La construction d'un modèle de système décisionnel nécessite de faire appel à la théorie générale des systèmes (Walliser, 1977, par exemple). Ainsi, un système décisionnel réalise des échanges avec son environnement, sous la forme d'entrées (informations, observations, etc) et de sorties (décisions); ces échanges sont sous l'influence de commandes (objectifs, rendez-vous avec les cultures, etc); il peut être décomposé en sous-systèmes en interaction (système d'information, système de décision proprement dit (Le Moigne, 1974) et il est luimême élément d'un système plus vaste (en interaction avec le système opérant). Un approfondissement de cette réflexion est en cours (Rellier, 1989).

La réalisation de modèles de systèmes décisionnels présente un triple intérêt potentiel :

- cognitif, pour représenter ce qu'on connaît (et, par différence, ce qu'on ne connaît pas) d'un processus de décision;

- prévisionnel, pour étudier, par simulation, certaines propriétés de tel ou tel système de décision;

- décisionnel, pour constituer des modules de systèmes informatiques d'aide à la décision, ce qui est bien notre objet ici.

Certains auteurs (Cerf et Sébillotte, 1988; Duru et al, 1988) ont proposé une représentation des processus de décision des agriculteurs (en fait, une esquisse de système décisionnel) en terme de modèle général à 3 composantes :

- un objectif (ou plusieurs) vers lequel tendent les décisions de l'agriculteur;

- un programme prévisionnel d'actions visant des objectifs intermédiaires (états-repères);

- un corps de règles d'adaptation qui définit la nature des décisions à prendre à chaque point intermédiaire, si l'objectif correspondant n'est pas atteint.

Ce modèle peut-il servir de cadre théorique pour les types de processus de décision dont nous avons développé des exemples, et qui n'en seraient alors que des cas particuliers?

Sur la mise en place de l'assolement, 2 points de vue sont possibles. Si le système biotechnique est un ensemble de peuplements végétaux, avec pour objectif, défini pour la campagne, d'assurer une certaine production, ou un certain revenu, alors le problème posé est celui de la configuration (c'est-à-dire la définition de la structure) du système. L'assolement de la campagne précédente n'est qu'une contrainte du problème, parmi d'autres. Rappelons ici que la résolution du problème précède complètement l'action (l'installation des peuplements) : on ne saurait alors parler de programme prévisionnel d'action ni d'états-repères du système biotechnique. Le modèle général ne s'applique pas. Mais si le système considéré est l'ensemble des parcelles cultivables et si l'objectif, défini à moyen terme, est d'assurer la continuité de la production (en nature, en quantité ou en revenu), alors nous pouvons appliquer le modèle général : le programme prévisionnel contient les orientations sur les cultures à pratiquer (fonction des terrains, des ressources, des marchés, etc), les objectifs intermédiaires sont, chaque année, des niveaux de marge brute, des états maximaux d'infestation des niveaux de charges de travail, etc, et une des règles d'adaptation est le processus de configuration de l'assolement évoqué plus haut. Les 2 problèmes sous-jacents sont alors la planification (au sens le plus large) d'activités et le contrôle du système biotechnique considéré.

Dans le problème de lutte contre' les adventices, le modèle général ne s'applique pas si on considère le processus ponctuel de décision : «Est-ce que je traite ? Si oui, comment ? Ou bien, est-ce que je mise sur un changement de culture ? Si oui, laquelle ?» Par contre, si on considère la conduite sur quelques années des parcelles cultivables, la lutte contre les adven- 
tices constitue, autant que la configuration d'assolement, une règle d'adaptation pour satisfaire le prochain objectif intermédiaire (à la fin de la prochaine campagne).

Ainsi, le modèle général s'applique aux processus de décision, éventuellement cycliques, où l'objectif assigné au système biotechnique est décomposable a priori, c'est-à-dire lorsqu'on peut prédéfinir une suite logique d'états-repères (éventuellement identiques) du système. En d'autres termes, on ne peut utiliser le modèle général que lorsque le problème est celui de la planification et du contrôle de l'évolution d'un processus. S'il s'agit, non plus de contrôler, mais de concevoir le système biotechnique, le modèle à itérations sera utilisé. En prenant l'exemple de la configuration de l'assolement, on voit que le modèle à itérations peut être implémenté seul, ou bien emboîté dans un modèle général de conduite de l'exploitation à moyen terme. Quant au modèle à étapes, on l'utilisera pour contrôler un système pour lequel l'objectif n'est pas décomposable dans le temps en sous-objectifs, la seule contrainte étant alors de maintenir les variables d'état du système dans un intervalle admissible (dans notre exemple : ne pas dépasser un degré d'infestation par les adventices). La figure 4 illustre ces distinctions.

\section{CONCLUSION}

Cette étude a montré qu'il est possible de décrire le comportement réel d'une classe particulière d'individus partageant le même problème de dé-
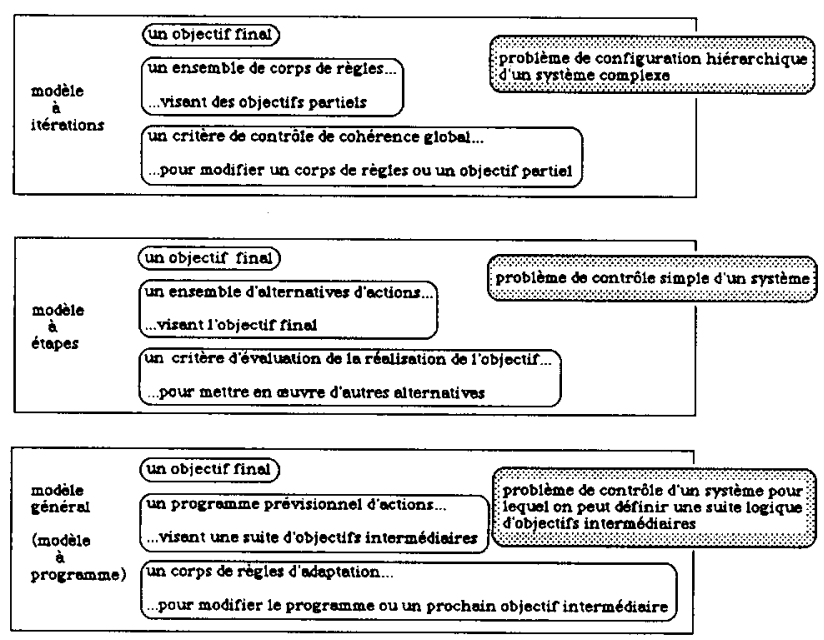

Fig 4. Trois types de modèles de la décision (modèlescadre). La case grisée contient la définition de la classe de problèmes concernée; les cases claires sont les éléments du modèle. cision et de généraliser ces descriptions sous forme de modèles. Les recherches sur le modèle général concourent au même résultat.

II apparaît alors qu'il existe un certain nombre de modèles-cadre (dont il faudrait sans doute compléter la liste). Chacun fournit la structure du système décisionnel pour un ensemble de problèmes de décision qui partagent la même définition générale (fig 4). La création d'un outil d'aide à la décision pour un problème donné est alors facilitée si on reconnaît qu'il existe déjà un modèle-cadre adapté. II suffit alors d'exprimer chaque composant du modèle-cadre dans les termes du domaine considéré : on construit alors un modèle-classe. Par exemple, à partir du modèle à itérations, on génèrera un modèleclasse de définition d'assolement, un modèleclasse de configuration de parc de matériel, un autre pour l'ensemble des troupeaux d'une exploitation, etc. Chaque modèle-classe est la structure d'un seul outil d'aide à la décision. Enfin, chaque agriculteur, ou chaque conseiller, pourra paramétrer le modèle-classe pour l'adapter à sa situation particulière, générant ainsi un modèle-instance, instance du modèle-classe, et conservant bien sûr la structure du modèlecadre adapté.

Une fois ces modèles de processus de décision écrits, il reste à les installer sur un ordinateur. On donne ainsi au logiciel d'aide à la décision le rôle d'un interlocuteur du décideur, possédant une autonomie de raisonnement et la capacité de comprendre interactivement la situation du décideur (objectifs, circonstances et préférences). Cela relève, selon le sens commun des mots, de l'intelligence artificielle. La discipline du même nom doit donc prendre une part du travail. Mémoire sélective (des actions passées), représentation des connaissances symboliques, heuristiques de résolution de problème, raisonnement hypothétique (en monde incertain), apprentissage, etc; autant d'aspects des raisonnements de l'agriculteur qui sollicitent I'IA [lire Bonnet (1984) pour une bonne introduction à la discipline]. Cependant l'illusion ne doit pas naître qu'un logiciel puisse montrer une intelligence au niveau du décideur humain. Facultés de perception, domaine de compétence, capacité d'apprentissage, etc resteront encore longtemps relativement limités. Dans ces conditions, les systèmes d'aide à la décision intelligents seront des systèmes spécialisés sur un problème particulier. Leur efficacité sera fonction des facultés de perception des utilisateurs et leur diffusion se heurtera, comme celle de systèmes classiques, au problème de la mainte- 
nance au cours du temps de la qualité des connaissances qu'ils contiennent.

\section{RÉFÉRENCES}

Attonaty JM, Soler LG (1988) Décision et incertitude en agriculture : les apports de la modélisation systémique et de l'intelligence artificielle. Rapport interne, INRA. Laboratoire d'économie et sociologie rurale, Grignon $6 \mathrm{p}$

Bailly C, Challine JF, Ferri HC, Gloess PY, Marchesin B (1987) Les Langages Orientés Objets. Cepadues, Toulouse, $233 p$

Blanchet A (1985) L'Entretien dans les sciences sociales. Bordas, Paris, $304 \mathrm{p}$

Bonnet A (1984) L'Intelligence artificielle. Promesses et réalités. InterÉditions, Paris, $271 \mathrm{p}$

Cerf $M$, Sebillotte $M$ (1988) Le concept de modèle général et l'analyse de la prise de décision. CR Acad Agric Fr 74, 4, 71-80

Charpenteau JL, Rellier JP (1980) Rotations culturales et modèles : utilisation de dispositifs expérimentaux. Actes du séminaire : Méthologie d'étude des systèmes de culture. CEE, Agrimed, Toulouse, 7-9 mai 1980, $20 \mathrm{p}$

Coulson RN, Saunders MC (1988) Types and characteristics of computer-based decison aids. Al Applications in Natural Resources Management 2, 39-43

De Kleer J (1986) An assumption-based truth maintenance system. Artificial intelligence 28, 127-162

Duru M, Papy F, Soler LG (1988) Le concept de modèle général et l'analyse du fonctionnement de l'exploitation agricole. CR Acad Agric Fr 74, 4, 81-93

France J, Thornley JHM (1984) Mathematical models in agriculture. Butterworths, Londres 37-68

Heurgon E (1985) Enjeux des systèmes interactifs d'aide à la décision (SIAD) et stratégie d'entreprise. Actes du Congrès : Systèmes Interactifs d'Aide à la Décision, Paris, 22 octobre 1985, ADI, ENA, CXP, 41-50
Howard RA (1968) The foundations of decision analysis. IEEE Transactions on Systems Sciences and Cybernetics SSC4 3, 1-9

Le Corfec F (1988) ZEA : Gestion des peuplements en culture de maïs. Actes du $2^{e}$ Congrès International DLG de l'Informatique : Les Systèmes d'Intelligence Artificielle en Agriculture, Frankfort, 19-22 juin 1988, 378-416

Le Moigne JL (1974) Les systèmes de décision dans les organisations. PUF, Paris

Marcaillou JC (1988) Le comportement de l'agriculteur lors de la prise de décision. Lutte contre les adventices. Mise en place de l'assolement. Mémoire École Supérieure d'Agriculture de PurpanToulouse. $122 p$

Marty JR, Cabelguenne M, Puech J (1984) Perspectives de valorisation d'un milieu par des assolements de grande culture : essai d'optimisation technico-économique. I. Élaboration d'un modèle de choix d'assolements. Agronomie 4, 871-884

Newell A, Simon HA (1963) GPS, a program that simulates human thought. In: Computers and Thought (Feigenbaum, Feldman, eds) McGraw-Hill, New York, 279-293

Pierret-Golbreich C (1989) Vers un système d'aide à la modélisation en biologie à base de schémas : EDORA. Actes des ges Journées : Les systèmes experts et leurs applications, Avignon, 29 mai-2 juin 1989, EC2, 35-54

Rellier JP (1989) Schéma fonctionnel général de la conduite d'un système de production. Rapport interne, INRA, Laboratoire d'intelligence artificielle, Toulouse, $12 p$

Richter MM (1988) Al-Concepts and OR-Tools in advanced decision-support systems. Decision Support Systems 4, 441-446

Rogers CR (1945) The non-directive method as a technique for social research. Am J Sociol 50, 279283

Walliser B (1977) Systèmes et modèles. Introduction critique à l'analyse de systèmes. Seuil, Paris, $248 \mathrm{pp}$ 